\title{
Revisión
}

\section{Proteoma urinario en la enfermedad renal diabética. Estado del arte}

\author{
Jorge Rico Fontalvo ${ }^{D}{ }^{1}$, Rodrigo Daza Anedo ${ }^{[D}{ }^{2}$, María Raad Sarabia (D) ${ }^{3}$, \\ Nehomar Pájaro Galvis (DD $\square^{3}$, Ariel Bello Espinosa ${ }^{(D)}$, Isabella Uparella \\ Gulfo (D) ${ }^{5}$, Christian Pérez Calvo (D) ${ }^{5}$, Alonso Pomares Lara (D) ${ }^{4,6}$, Zulay \\ Mondol Almeida (D) ${ }^{3}$, Oscar Vergara-Serpa ${ }^{(D)}{ }^{3}$, Carlos Berrocal-Martinez ${ }^{\text {(D) }}{ }^{3}$, \\ Luis Adolfo Collazos Torres (D)7, e Karen Ruiz Pastrana (D) 8 \\ ${ }^{1}$ Comité de Nefrodiabetes, Asociación Colombiana de Nefrología e Hipertensión Arterial, Medellín, Colombia. \\ ${ }^{2}$ Comité de Nefrodiabetes, Asociación Colombiana de Nefrología e Hipertensión Arterial, Cartagena, Colombia. \\ ${ }^{3}$ Facultad de Ciencias de la Salud, Universidad del Sinú, Cartagena, Colombia. ${ }^{4}$ Departamento de Medicina \\ Interna, Universidad de Cartagena, Cartagena, Colombia. ${ }^{5}$ FDepartamento de Medicina Interna, Universidad de \\ Cartagena, Cartagena, Colombia. ${ }^{6}$ Departamento de Medicina Interna, Clínica San José de Torices, Cartagena, \\ Colombia. ${ }^{7}$ Facultad de Medicina, Universidad de Sucre, Sincelejo, Colombia. ${ }^{8}$ Programa de rutas integrales, \\ Promoción Salud, Medellín, Colombia.
}

Cómo citar: Rico Fontalvo J, Daza Arnedo R, Raad Sarabia M, Pájaro Galvis N, Bello Espinosa A, Uparella Gulfo. Proteoma urinario en la enfermedad renal diabética. Estado del arte. Rev. Colomb. Nefrol. 2021, 8(3), e546. https://doi.org/10.22265/acnef.8.3.546

Recibido: 28/Dic/2020 Aceptado: 16/Mar/2021 Publicado: 18/Ago/2021

\section{Resumen}

La enfermedad renal diabética (ERD) es la principal complicación microvascular de los pacientes con diabetes mellitus; esta es una entidad que genera un aumento significativo en la mortalidad de origen cardiovascular de este grupo de pacientes, aunque su diagnóstico temprano impacta de forma significativa en la evolución a enfermedad renal terminal y, por lo tanto, en la mortalidad. Ladetección de albuminuria en la orina y el deterioro de la tasa de filtración glomerular estimada son las principales técnicas diagnósticas que se utilizan en la práctica clínica para establecer la presencia de ERD; sin embargo, estas tienen limitaciones y por lo tanto es importante resaltar que el daño renal suele ser irreversible una vez están presentes. Durante los últimos años, numerosos estudios se han enfocado en detectar nuevos biomarcadores para detectar ERD y es aquí donde aparece como nueva herramienta la proteómica urinaria, una tecnología emergente que permite identificar en una muestra de orina proteínas que sugieren la presencia de esta enfermedad de manera temprana. Asimismo, el descubrimiento de biomarcadores basados en proteómicos representa una estrategia novedosa para mejorar el diagnóstico, pronóstico y tratamiento de la

$\checkmark$ Correspondencia: Transversal 54 41-117, Cartagena, CO. +57 3008233570. neho94@hotmail.com 
nefropatía diabética; sin embargo, los enfoques basados en la proteómica aún no están disponibles en la mayoría de los laboratorios de química clínica.

Palabras clave: proteoma; nefropatías diabéticas; diabetes mellitus.

\title{
Urinary proteome in diabetic kidney disease: state of the art
}

\begin{abstract}
Diabetic kidney disease (DKD) is the main microvascular complication in patients with diabetes mellitus; it is an entity that generates a significant increase in mortality of cardiovascular origin in this group of patients, although its early diagnosis has a significant impact on the evolution to end-stage kidney disease and, therefore, on mortality. The detection of albuminuria in urine and the deterioration of the estimated glomerular filtration rate are the main diagnostictechniques that are used in clinical practice to establish the presence of DKD; however, they have limitations and therefore it is important to note that kidney damage is usually irreversible once they are present. Over the last few years, numerous studies have focused on the discovery of new biomarkers to detect DKD and this is where the urinary proteomics appears as a new tool, an emerging technology that allows the identification of proteins in a urine sample that strongly suggest the early presence of this disease. Likewise, the discovery of proteomic-based biomarkers represents a novel strategy to improve the diagnosis, prognosis and treatment of diabetic nephropathy; however, proteomics-based approaches are not yet available in the majority of clinical chemistry laboratories.
\end{abstract}

Keywords: Proteome; Diabetic Nephropathies; Mellitus diabetes.

\section{Introducción}

La diabetes mellitus (DM) se ha posicionado como una de las principales causas de mortalidad cardiovascular en las últimas décadas dada su asociación con el síndrome metabólico, la obesidad y la aparición de complicaciones macro y microvasculares que impactan directamente en la calidad de vida de los pacientes y en la letalidad de la enfermedad; además, dada su alta prevalencia a nivel mundial, constituye un problema de salud pública [1].

La enfermedad renal diabética (ERD) constituye, al día de hoy, la complicación microvascular más común de los pacientes con DM; además, a lo largo de losaños se ha convertido en la primera causa de enfermedad renal crónica (ERC) a nivel mundial, teniendo un alto impacto en la mortalidad temprana de los pacientes que padecen esta dualidad [2]. La ERC se caracteriza por un patrón histopatológico de engrosamiento en la membrana basal glomerular, 
expansión de la matriz mesangial, glomeruloesclerosis nodular e hialinosis arteriolar, lo que conduce a una pérdida progresiva de la masa renal funcional con la consecuente disminución de la tasa de filtrado glomerular (TFG) y perdida de la función renal [3].

La ERD suele aparecer en los siguientes 10 a 15 años posteriores al diagnóstico de la DM y resulta de la interacción compleja de factores genéticos, ambientales, metabólicos, hemodinámicos, inflamatorios y profibróticos que generan un efecto deletéreo en la estructura y la función del riñón [4]. De esta forma, el estado de hiperglucemia permanente y de inflamación crónica en los individuos que padecen DM genera daño en los tejidos y compromiso endotelial que lesiona diversos órganos, favoreciendo así la modificación de moléculas a nivel tisular con la consecuente aparición de los productos avanzados de glicosilación que afectan la estructura y la función de la matriz extracelular con una elevada producción de radicales libres y radicales superóxidos que desencadenan la lesión microvascular y que, a nivel renal, conduce a la ERD [5].

El compromiso renal de la DM, más allá del daño endotelial, es generado, en primer lugar, por una activación del sistema renina-angiotensina-aldosterona a nivel tubular que induce la producción de diversos factores inflamatorios y profibroticos [6]; y, en segundo lugar, por una alteración en el mecanismo deretroalimentación túbulo-glomerular, lo que finalmente conduce a un estado de hiperfiltración glomerular, hipertrofia renal y daño a nivel mesangiocapilar [7].

La presentación clásica de la ERD incluye un aumento progresivo de la proteinuria hasta alcanzar, en algunos casos, rangos nefróticos; una disminución del filtrado glomerular, y alteraciones en el sedimento urinario (cilindros, proteinuria, glucosuria, hematuria). Las herramientas actuales para el diagnóstico de ERD incluyen la evaluación constante de la TFG y de la tasa de excreción de albumina urinaria; sin embargo, se ha demostrado que ambos marcadores carecen de la capacidad de predecir de manera confiable la aparición y progresión de la enfermedad [8], por lo que en la era actual de las tecnologías de alto rendimiento los investigadores han dedicado sus esfuerzos a la búsqueda y desarrollo de biomarcadores no invasivos que permitan la detección cada vez más temprana de alteraciones a nivel renal secundarias a ERD.

La proteómica urinaria ha emergido en los últimos años como uno de los nuevos objetivos en cuanto a biomarcadores para el diagnóstico inicial de la ERD; esta comprende el conjunto de proteínas expresadas en una muestra de secreción biológica. 
Dado que el proteoma urinario proviene en un $70 \%$ del tracto urogenital, es probable que represente el biomarcador más estable en el riñón, por lo cual se ha estudiado en los últimos años con el objetivo de identificar, por un lado, marcadores iniciales de progresión de enfermedad renal y, por el otro,herramientas diagnosticas o para medir la respuesta a la terapia médica instaurada.

En esta revisión de la literatura, luego de un breve resumen de la fisiopatología de la ERD y las estrategias actuales de diagnóstico, se revisan los hallazgos más recientes sobre el proteoma urinario en el contexto de la ERD.

La ERD constituye una causa importante de enfermedad renal progresiva y permanente y es la complicación microvascular más conocida de la diabetes de larga evolución [9]. Hoy en día esta es una de las principales causas de disminución de la esperanza de vida de los pacientes diabéticos, e incluso en los primeros años de su diagnóstico contribuye a un aumento importante en el riesgo de enfermedad y muerte cardiovascular.

El mal control glucémico es el principal factor de riesgo para desarrollar ERD; sin embargo, la presencia de obesidad mórbida, el bajo peso al nacer y los factores genéticos de susceptibilidad se han descrito como factores que aumentan el riesgo de deterioro renal en pacientes con DM tipo 1 y tipo 2 [10]. Por otra parte, la TFG juega un papel importante en el desarrollo de lesión renal, pues los pacientes diabéticos con hiperfiltración glomerular tienen mayor riesgo de desarrollar ERD establecida con albuminuria con un riesgo mayor si la TFG inicial es $>150$ $\mathrm{mL} / \mathrm{min}[11,12]$.

\section{Mecanismos fisiopatológicos de la ERD}

La manifestación clínica más detectable de la ERD es la presencia de albuminuria grado I; además, ante la ausencia de una intervención adecuada y oportuna, el 50\% de los pacientes progresan a albuminuria grado III, lo que se asocia con un mayor deterioro de la función renal respecto a aquellos pacientes sin presencia de albuminuria [13]. La incidencia de albuminuria en pacientes con DM tipo 1 y tipo 2 es de alrededor del $30 \%$ y depende en gran medida del cumplimiento de la medicación y del control glucémico en los diabéticos tipo 1, y de la presencia de hipertensión arterial controlada concomitante en los diabéticos tipo 2; sin embargo, más allá del control glucémico, otros factores que influyen en el desarrollo de albuminuria incluyen edad avanzada, creatinina elevada y mal control de hemoglobina glucosilada. 
La hiperglucemia conduce a un aumento consecuente de la cantidad de glucosa filtrada a través de la barrera de filtración, lo que a su vez induce al aumento de la reabsorción de glucosa en el túbulo proximal; este proceso implica una inducción en la expresión de trasportadores de glucosa con aumentos masivos de los procesos de trasporte a nivel celular, elevando así el consumo de energía en las células del túbulo proximal y, consecuentemente, los requerimientos de oxígeno en la corteza renal y la medula externa; asimismo, la limitación para suplir la demanda de oxígeno en la nefrona induce una isquemia relativa con una producción de la expresión de marcadores de estrés celular como la lipocaleina asociada a gelatinasa de neutrófilos (NGAL) y la molécula de lesión renal (KIM- 1) [14].

Por otra parte, el aumento en la expresión del cotransportador SLGT2 estimula la recuperación de sodio de forma masiva en el túbulo proximal reduciendo la concentración de dicho ion en el túbulo contorneado distal y en la macula densa con la consecuente inactivación del mecanismo de retroalimentación tubuloglomerular, lo que induce la vasodilatación de la arteriola aferente y libera la renina que estimula la vasoconstricción de la arteriola eferente; estos efectos hemodinámicos conducen a una hiperfiltración e hipertensión glomerular.

De igual forma, el aumento de la función de filtración en el túbulo proximal conduce a su alargamiento, lo que contribuye con el proceso de hipertrofia renal que caracteriza a la ERD [15].

En cuanto a los efectos tardíos de la hiperglucemia a nivel renal, la disfunción endotelial y el daño en la microvasculatura constituyen la base fundamental de los mecanismos patógenos involucrados en el desarrollo de la ERD; en este sentido, el aumento de la permeabilidad vascular, la inflamación y la desregulación del tono vascular son la causa y la consecuencia de que los pacientes cursen con ERD [16].

En el riñón, ante la presencia de ERD, la disfunción endotelial resulta en un daño de la barrera de filtración debido al aumento de la permeabilidad vascular; además, la lesión a nivel de los podocitos genera la consecuente aparición de albuminuria, considerándose esta última, en el contexto de la DM, como un marcador de disfunción endotelial sistémica que es reconocido como un factor de riesgo independiente para la mortalidad cardiovascular. En la figura 1 se resumen los mecanismos fisiopatológicos de la ERD.

Clásicamente se han descrito cuatro tipos de lesiones glomerulares en los pacientes que desarrollan ERD: glomeruloesclerosis nodular (lesiones de Kimmesltiel-Wilson); glomeruloesclerosis difusa; gota capsular con depósitos de material eosinofílico entre la membrana 


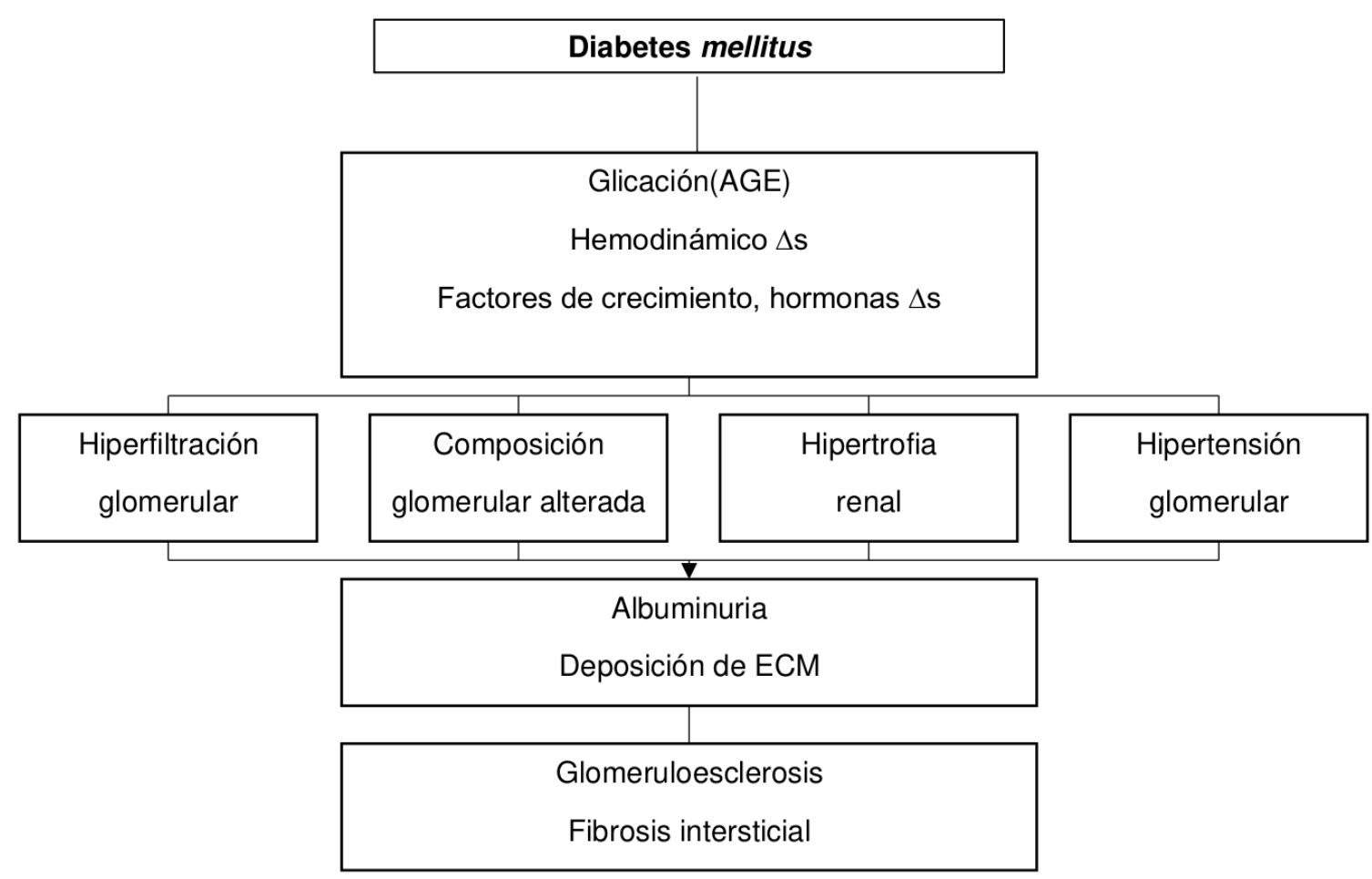

Figura 1: Mecanismos fisiopatológicos de la enfermedad renal diabética. ECM: matriz extracelular; AGE: productos finales de glicación avanzada.

Fuente: elaboración con base en Umanath \& Lewis [4].

basal glomerular (MBG) y la célula epitelial, y depósitos extracapilares de fibrina; además, se ha establecido que junto a las lesiones a nivel glomerular se genera una afectación vascular y lesiones túbulo-intersticiales con predominio de fibrosis [17].

Durante el progreso natural de la ERD, que relaciona tanto las manifestaciones clínicas como las lesiones morfológicas y los cambios funcionales a nivel renal, se suelen identificar cinco fases: 1) hipertrofia renal e hiperfiltración glomerular, 2) lesión renal sin signos clínicos, 3) nefropatía incipiente, 4) nefropatía diabética establecida y 5) enfermedad renal terminal. Sin embargo, es importante reconocer que la evolución clínica de esta patología no es lineal y que, además, en la literatura se ha descrito que cerca del $30 \%$ de los pacientes con DM no presentan albuminuria, lo que se conoce como "fenotipo no-albuminurico" [18]. Los cambios fisiopatológicos y las manifestaciones clínicas de cada fase de la ERD se resumen en la figura 2.

\section{Diagnóstico temprano de ERD}

Las implicaciones en el riesgo cardiovascular de la ERD y el conocimiento del impacto en la mortalidad que tiene esta complicación en los individuos con DM han conducido en los últimos años a una optimización en las estrategias de detección temprana de esta patología. 
La albuminuria es el marcador inicial más utilizado en la actualidad para el diagnóstico de ERD, y la American Diabetes Association recomienda la detección anual de albumina urinaria y tasa de filtración glomerular estimada (eTFG) en pacientes con DM tipo 1 con un diagnostico mayor a 5 años, en todos los pacientes con DM tipo 2 desde el momento del diagnóstico y en todos los pacientes con hipertensión arterial comórbida [9].

La detección de albumina en orina se puede realizar mediante tres métodos: recolección de muestra de orina en 24 horas, recolección programada de orina (durante 4 horas o durante la noche) o medición de la relación albumina/creatinina en momentos aleatorios [19], siendo esta última la técnica más utilizada (preferiblemente medida en una muestra de la mañana); sin embargo, debido a la alta variabilidad interdiaria, los resultados anormales deben ser confirmados por al menos dos muestras patológicas en un periodo de 3 a 6 meses. Se define

\begin{tabular}{|c|c|c|c|}
\hline $\begin{array}{c}\text { Etapas de } \\
\text { efropatía diabética }\end{array}$ & Hipertrofia renal & incipiente & abierta \\
\hline
\end{tabular}

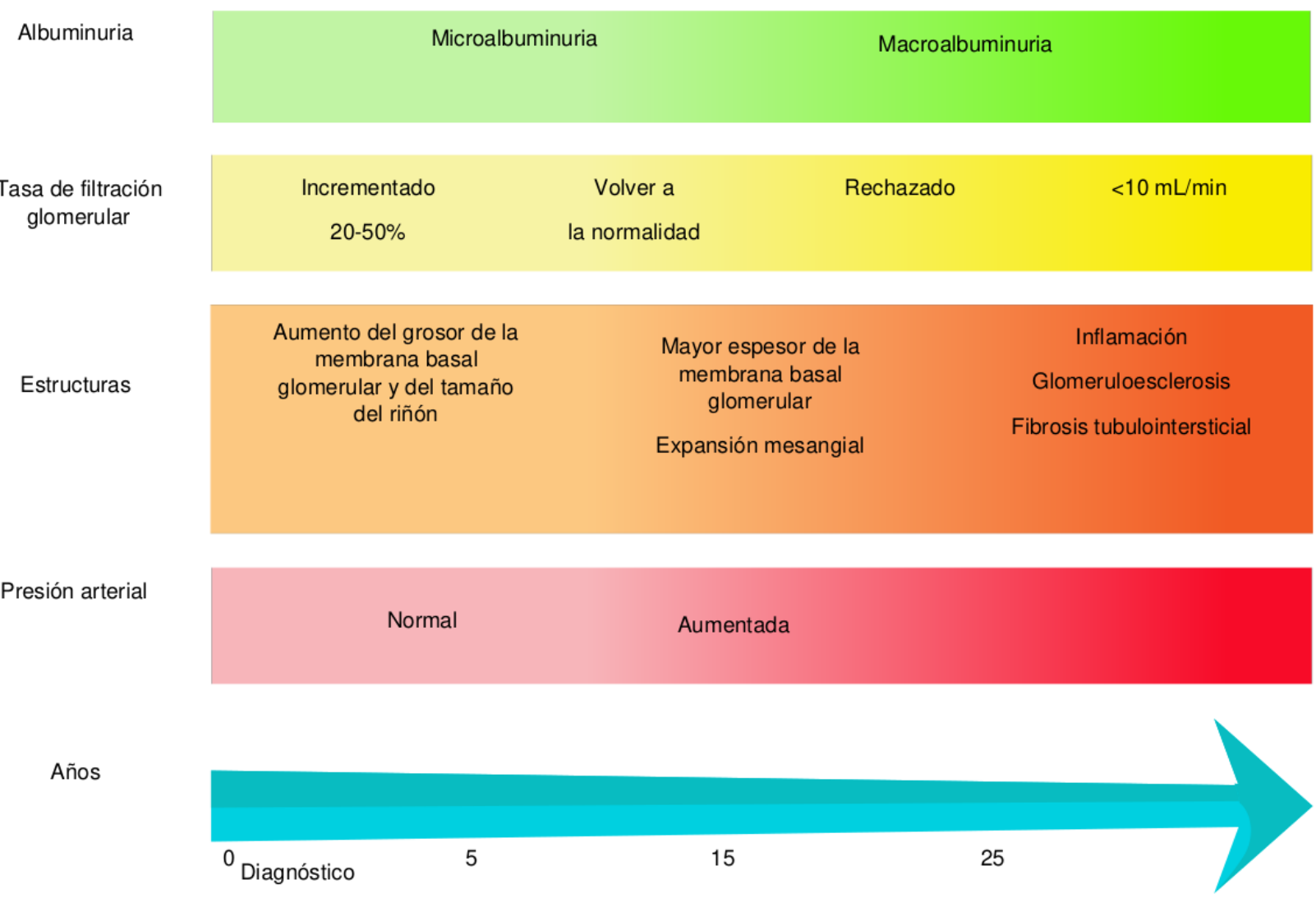

Figura 2: Historia natural de enfermedad renal diabética.

Fuente: elaboración con base en Umanath \& Lewis [4]. 
como anormal más de $30 \mathrm{mg}$ de albumina en una muestra al azar o más $30 \mathrm{mg}$ de albumina por gramo de creatinina [20].

Los estudios para determinar la presencia de ERD también deben incluir la medición de creatinina sérica y la eTFG, además el diagnostico se realizaclínicamente cuando un paciente tiene evidencia de ERC y ninguna otra etiología primaria. El desarrollo de síndrome nefrótico, la ausencia de retinopatía diabética, la aparición repentina de un descenso en la eTFG, la presencia de hipertensión refractaria, el sedimento urinario activo y el aumento brusco de la albuminuria deben hacer considerar la evaluación de causas de enfermedad renal no relacionadas con la diabetes [21].

Por otra parte, la ecografía renal es una prueba morfológica básica que debe solicitarse en todos los pacientes en quienes se sospeche ERD, pues permite establecer el tamaño renal y la diferenciación parenquimatosa, y descartar otras causas de enfermedad renal como la obstrucción del tracto urinario [22].

A pesar de lo anterior, las pruebas disponibles en la actualidad para el cribado de la ERD tienen una serie de limitaciones que han conducido a que se investiguen nuevos biomarcadores que representan diversos procesos moleculares que permiten el diagnóstico temprano y oportuno de la ERD, incluso en estadios en los cuales el paciente aún permanece con valores normales de albumina en orina y ausencia o deterioro leve de la eTFG.

\section{Proteoma urinario}

El termino proteoma se utiliza para definir el estudio de proteínas a gran escala y, dado que los niveles de proteínas cambian bajo diversas condiciones fisiológicas, su análisis también se utiliza para dilucidar el mecanismo de la enfermedad y permite identificar biomarcadores tempranos para el diagnóstico de ERD [23]; así mismo, el estudio de las proteínas puede utilizarse como herramienta diagnosticapara la detección y estadificación de enfermedades, o para predecir el resultado terapéutico, la respuesta a una intervención y la supervivencia de un manejo determinado.

En síntesis, el método proteómico de análisis de proteínas permite realizar una evaluación rápida del proteoma, que es el inventario completo de proteínas expresadas en una muestra biológica; con este método, las muestras biológicas (orina, plasma o suero) se pueden analizar sistemáticamente para identificar, cuantificar y discernir la función de todas las proteínas observables $[24,25]$. La orina, al ser un fluido biológico susceptible de ser recogido de forma 
no invasiva y encontrándose fácilmente disponible en la mayoría de centros hospitalarios, se ha convertido en una muestra ideal y valiosa para el estudio de biomarcadores de diversas enfermedades clínicas.

La orina es producida por el riñón para eliminar los productos de desecho del plasma; cerca del $30 \%$ de las proteínas de la orina se origina en las proteínas plasmáticas, mientras que el $70 \%$ restante proviene del riñón y del tracto urinario; por lo tanto, el estudio del proteoma urinario proporciona importantes biomarcadores que reflejan directamente las funciones del riñón y los órganos relacionados [26]. Como una de las fuentes para la detección de nuevos biomarcadores, la orina ha mostrado varias ventajas: puede tolerar un grado de cambio mayor que las proteínas plasmáticas, lo que facilita su detección; es de fácil recolección, y aunque su muestra es menos compleja que el plasma tiene un contenido de proteínas, péptidos y aminoácidos que no se han descubierto en muestras plasmáticas [27].

Los primeros informes del uso del proteoma urinario se hicieron hace aproximadamente 15 años; en estos se presentó dicho análisis como una herramienta útil en el diagnóstico de ERC y su etiología [28,29]; además, en estudios posteriores, mediante esta tecnología, se logró identificar biomarcadores específicos para nefropatía por IgA y nefropatía membranosa [30]. Desde entonces el esfuerzo se enfocó en el estudio de la muestra de orina para identificar proteínas en diversas entidades clínicas como la ERD [31] y las vasculitis asociadas a anticuerpos anticitoplasma de neutrófilos [32].

La recolección de la muestra de orina no requiere de una técnica en especial para el estudio, aunque se prefiere la elección de la muestra de mitad de la mañana para eliminar el riesgo de contaminación. Las muestras pueden tener un crecimiento excesivo de bacterias durante el almacenamiento, por lo que se sugiere administrar conservantes para evitar la proliferación bacteriana después de la recolección; la temperatura ideal varía según el tiempo en el que se analizaran las muestran, sin embargo se recomienda que sea de alrededor de $4^{\circ} \mathrm{C}$ para evitar la degradación de proteínas [33].

En cuanto a las diferentes tecnologías para el estudio proteómico, se considera que una técnica analítica ideal utilizada para el análisis de proteomas debe tener capacidades de alta resolución, alta sensibilidad y alto rendimiento de separación e identificación de proteínas; algunas de las técnicas más usadas son la electroforesis bidimensional (2-DE), la electroforesis en gel de diferencia bidimensional (2-D DIGE), la cromatografía líquida acoplada a espectrometría de masas en tándem (LC-MS / MS), la electroforesis capilar acoplada aespectrometría de 
masas (CE-MS), los microarrays de inmunoensayos de espectrometría de masas y la tecnología de microfluidos (figura 3).

\section{Proteoma urinario en ERD}

Como se mencionó antes, el diagnóstico de la ERD se basa en la detección de albuminuria y de un descenso en la eTFG; sin embargo, se ha demostrado que cuando estos marcadores empiezan a ser detectados ya existe un daño renal instaurado, además se han descrito casos de ERD no-albuminurica [34]. Por todo esto, en los últimos años el estudio del proteoma urinario se ha venido desarrollando con el fin de identificar biomarcadores tempranos que permitan realizar intervenciones previas a la instauración de daño de órgano blanco y, por tanto, previo a la aparición de albuminuria y de deterioro de eTFG.

La técnica proteómica más utilizada para el cribado de ERD es la 2-DE, la cual separa las proteínas por $\mathrm{pH}$ diferencial en la primera dimensión y por masas moleculares diferenciales en la segunda dimensión; esta tecnología permite caracterizar el proteoma renal, incluidos los proteomas de la corteza renal, las células glomerulares y las células epiteliales tubulares [35]. En segundo lugar suele recomendarse la combinación en línea de electroforesis capilar/espectrometría de masas por electropulverización (CE/MS) para un enfoque rápido
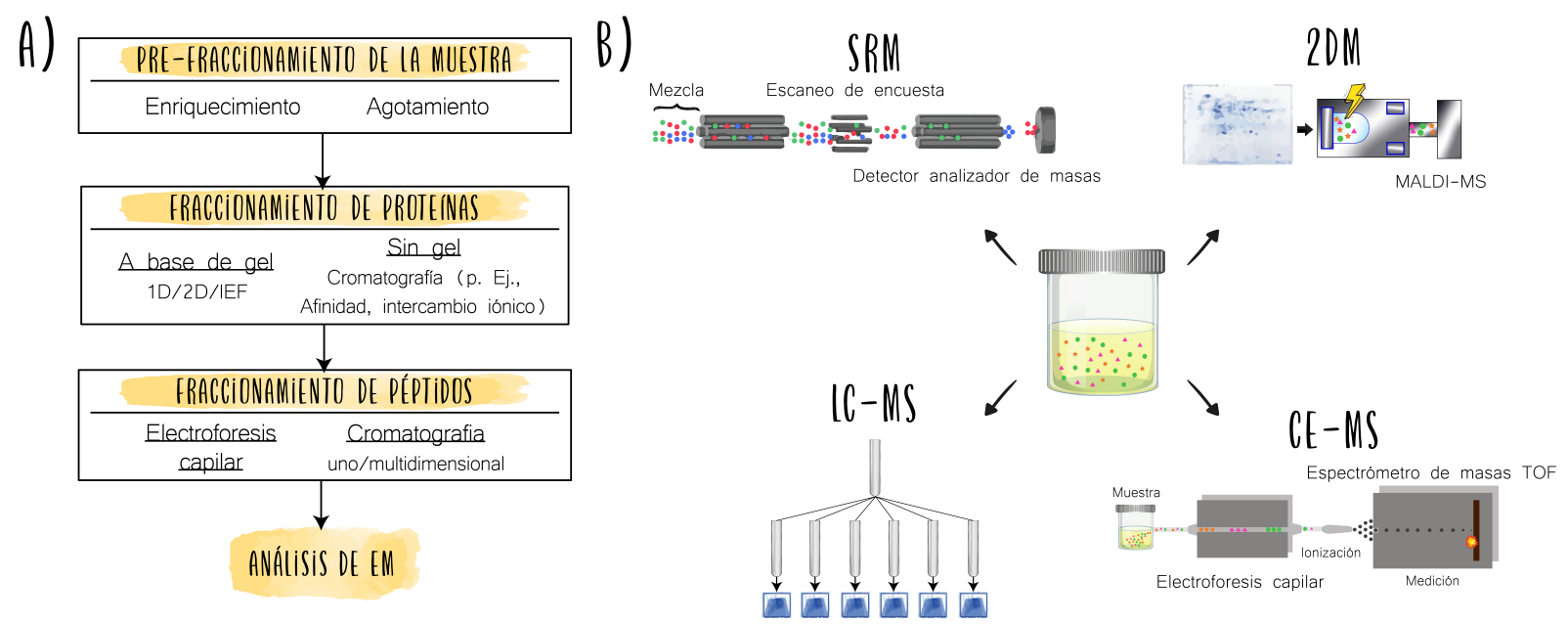

Figura 3: Protocolo de trabajo en proteómica urinaria. A) Técnicas de prefraccionamiento, fraccionamiento de proteínas y fraccionamiento de péptidos; B) Plataformas proteómicas aplicadas de forma rutinaria.

SRM: monitoreo de reacción seleccionado; MS: espectrometría de masa, 2DM: electroforesis bidimensional; LC-MS: cromatografia liquida - espectrometría de masas; CE-MS: electroforesis capilar acoplada a espectrometría de masas.

Fuente: elaboración con base en Filip et al. [23]. 
(aproximadamente 45 minutos por muestra), sensible y automatizado en el análisis de las muestras de orina [36].

Hoy en día existen numerosos estudios que han descrito la utilidad del proteoma urinario en el diagnóstico temprano de la ERD y en los cuales se han identificado biomarcadores asociados con la enfermedad antes de la aparición de albuminuria, incluidos fragmentos específicos de colágeno, citocinas proinflamatorias, $\beta 2$-Microglobulina ( $\beta 2-M G)$ y proteína de unión al retinol (RBP). En 2012, por ejemplo, Zürbig et al. [36] examinaron muestras de orina de una cohorte longitudinal de pacientes diabéticos tipo 1 y 2 utilizando un clasificador de biomarcadores de ERC generado previamente para evaluar los péptidos de orina recolectada en busca de signos de ERD, y encontraron que la aplicación de este clasificador en orina de sujetos normoalbuminúricos hasta 5 años antes del desarrollo de albuminuria permitió detectar precozmente la progresión posterior a macroalbuminuria; además, los autores evidenciaron que los fragmentos de colágeno fueron biomarcadores prominentes 3-5 años antes del inicio de la albuminuria con un descenso en estos poco antes que la excreción de albúmina comience a aumentar.

Por otra parte, y como validación del modelo de clasificación de proteoma urinario, Papale et al. [37] evaluaron los perfiles proteómicos de la orina generados por espectrometría de masas con el objetivo de aislar un conjunto de biomarcadores que pudieran identificar de manera confiable la ERD y establecer una estricta correlación con los diferentes patrones de lesión renal, y encontraron que el modelo de clasificación identificó correctamente el $75 \%$ de los pacientes con normoalbuminuria, el $87,5 \%$ de aquellos con albuminuria grado I y el $87,5 \%$ de aquellos con ERD cuando se aplicó a un conjunto de pruebas ciegas. De igual manera, este modelo fue capaz de diferenciar de forma fiable la ERD de la ERC no diabética, tanto en pacientes diabéticos como no diabéticos. Los autores también lograron identificar entre los mejores predictores del modelo de clasificación a dos proteínas: la ubiquitina y la $\beta 2-\mathrm{MG}$ [37].

De forma más reciente, Roscioni et al. [38] evaluaron el valor de una puntuación de riesgo basada en la proteómica urinaria (clasificador) para predecir el desarrollo y la progresión de la albuminuria en pacientes diabéticos tipo 2, para lo cual se construyó un modelo que permitiera establecer la progresión de la albuminuria utilizando un clasificador proteómico basado en 273 péptidos urinarios; este clasificador proteómico se asoció de forma independiente con la transición a albuminuria grado II o grado III y el clasificador predijo el desarrollo y la progresión de la albuminuria, además del deterioro de la eTFG [38]. En la tabla 1, se resumen los principales biomarcadores que se han descrito para ERD basados en el proteoma urinario. 
Tabla 1: Biomarcadores descritos para ERD.

\begin{tabular}{|c|c|c|c|}
\hline Biomarcador & Población de estudio & Principales hallazgos & Referencia \\
\hline $\begin{array}{c}\text { Fragmentos } \\
\text { específicos de } \\
\text { colágeno } \\
\text { (colágeno tipo IV) }\end{array}$ & $\begin{array}{c}82 \text { pacientes } \\
\text { diabéticos tipo } 2 \text { y } 205 \\
\text { controles }\end{array}$ & $\begin{array}{c}\text { El colágeno tipo IV urinario en } \\
\text { pacientes diabéticos tipo } 2 \text { fue } \\
\text { mayor que en los controles } \\
(7,7 \pm 4,5 \mu \mathrm{g} / \mathrm{g} \text { de creatinina } \\
\text { frente a } 3,0 \pm 1,7 \mu \mathrm{g} / \mathrm{g} \text { de } \\
\text { creatinina) }\end{array}$ & [36] \\
\hline $\begin{array}{c}\beta 2 \text {-Microglobulina } \\
(\beta 2-\mathrm{MG})\end{array}$ & $\begin{array}{c}946 \text { pacientes con } \\
\text { diabetes mellitus tipo } 2\end{array}$ & $\begin{array}{c}\text { Los niveles urinarios de } \beta 2-\mathrm{MG} \\
\text { fueron más altos en pacientes } \\
\text { con complicaciones macro y/o } \\
\text { microvasculares en } \\
\text { comparación con aquellos que } \\
\text { no las tenían. Los pacientes con } \\
\text { hipertensión asociada tenían } \\
\text { niveles urinarios más altos de } \\
\beta 2-\mathrm{MG} \text {, independientemente de } \\
\text { si las complicaciones estaban } \\
\text { presentes o no }\end{array}$ & {$[37]$} \\
\hline $\begin{array}{l}\text { Proteína de unión } \\
\text { al retinol (RBP) }\end{array}$ & $\begin{array}{c}59 \text { pacientes } \\
\text { diabéticos tipo } 1(11 \\
\text { con albuminuria grado } \\
\text { I y } 48 \text { con normal } \\
\text { EAU) y } 40 \text { sujetos } \\
\text { de control }\end{array}$ & $\begin{array}{c}\text { La RBP se excretó en } \\
\text { cantidades significativamente } \\
\text { mayores en los pacientes } \\
\text { diabéticos en comparación con } \\
\text { los controles. Los pacientes } \\
\text { diabéticos con albuminuria } \\
\text { tienen mayor excreción urinaria } \\
\text { de RBP que los pacientes } \\
\text { diabéticos con EAU normal }\end{array}$ & [38] \\
\hline KIM-1 & $\begin{array}{l}\text { Pacientes diabéticos } \\
\text { tipo } 1 \text { ( } 58 \text { con } \\
\text { albuminuria grado I, } \\
45 \text { con albuminuria } \\
\text { grado II y } 45 \text { con } \\
\text { albuminuria grado III) } \\
\text { y } 55 \text { control }\end{array}$ & $\begin{array}{l}\text { La excreción urinaria de KIM-1 } \\
\text { fue mayor en todos los grupos } \\
\text { de diabéticos que en el grupo } \\
\text { de control, sin diferencias entre } \\
\text { los grupos de diabéticos }\end{array}$ & [39] \\
\hline
\end{tabular}

EAU: Excreción urinaria de albúmina; KIM-1: Kidney Injury Molecule-1.

Fuente: elaboración propia.

Como ya se mencionó, diversos estudios se han enfocado en evaluar la utilidad del proteoma urinario como técnica de diagnóstico temprano de la ERD; sin embargo, dada la escasa disponibilidad de dicha tecnología, esta es una práctica no estandarizada de forma rutinaria en los diversos escenarios clínicos, aunque se debe reconocer que en un futuro podría contri- 
buir de gran manera a la detección temprana de pacientes con ERD, sobre todo aquellos con "fenotipo no- albuminurico".

\section{Conclusiones}

La ERD es una de las complicaciones microvasculares más comunes que impactan en la mortalidad del paciente diabético; por lo tanto, diagnosticarla de manera temprana es de suma importancia para evitar su progresión y desenlaces fatales.

La presencia de albuminuria sigue siendo la técnica de preferencia para el cribado de la ERD; sin embargo, el descubrimiento de biomarcadores urinarios basados en la proteómica representa una nueva estrategia para mejorar el diagnóstico, pronóstico y tratamiento de la enfermedad en etapas tempranas y previas a la instauración de un daño renal irreversible.

Aunque las muestras de suero se pueden usar para análisis proteómicos, la orina se ha convertido, cada vez más, en una fuente importante para la evaluación de biomarcadores basados en proteínas debido a la facilidad de recolección y manejo preanalítico, y a la estabilidad de los péptidos en esta sustancia.

Si bien las técnicas para el estudio del proteoma urinario no se encuentran disponibles en todos los escenarios clínicos, se ha sugerido que los enfoques basados en la proteómica son muy prometedores como base para las nuevas pruebas de diagnóstico y como medio para comprender mejor la patogénesis o la evolución de la ERD, pues ofrece el desarrollo de predictores novedosos, no invasivos y tempranos de esta enfermedad.

\section{Consideraciones éticas}

Los autores declaran que los procedimientos seguidos se realizaron conforme a las normas éticas del comité de experimentación humana responsable y de acuerdo con lo establecido por la Asociación Médica Mundial en la Declaración de Helsinki; que han seguido los protocolos de su centro de trabajo sobre la publicación de datos de pacientes,y que han obtenido el consentimiento informado de los pacientes y/o sujetos referidos en el artículo.

\section{Contribución de los autores}

Jorge Rico Fontalvo, Rodrigo Daza Arnedo, María Raad Sarabia, Nehomar Pájaro Galvis, Ariel Bello Espinosa, Christian Pérez Calvo: Redacción del manuscrito. Isabella Uparella Gulfo, 
Alonso Pomares Lara, Zulay Mondol Almeida, Oscar Vergara Serpa, Carlos Berrocal Martínez: Gráficas y tablas. Luis Collazos Torres, Karen Ruiz Pastrana: Busqueda avanzada y selección de fuentes bibliográficas

\section{Conflicto de intereses}

Ninguno declarado por los autores.

\section{Financiación}

Ninguna declarada por los autores.

\section{Referencias}

[1] American Diabetes Association. Standars of Medical Care in Diabetes - 2020 Abridged for Primary Care Providers. Clin Diabetes C. 2020;38(1):10-38. https://dx.doi.org/10.2337/ cd20-as01. $\uparrow$ Ver página 2

[2] Chan GCW, Tang SCW. Diabetic nephropathy: Landmark clinical trials and tribulations. Nephrol Dial Transplant. 2016;31(3):359-68. https:/dx.doi.org/10.1093/ndt/gfu411. 个Ver página 2

[3] Anders HJ, Huber TB, Isermann B, Schiffer M. CKD in diabetes: Diabetic kidney disease versus nondiabetic kidney disease. Nat Rev Nephrol. 2018;14(6):361-77. https://x.doi.org/ 10.1038/s41581-018-0001-y. 个Ver página 3

[4] manath K, Lewis JB. Update on Diabetic Nephropathy: Core Curriculum 2018. Am J Kidney

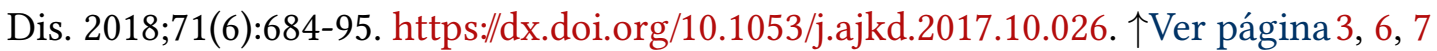

[5] Wada J, Makino H. Inflammation and the pathogenesis of diabetic nephropathy. Clin Sci (Lond). 2013;124(3):139-52. https://dx.doi.org/10.1042/CS20120198. 个Ver página 3

[6] Duran-Salgado MB, inflammation. Rubio-Guerra World J AF. Diabetic nephropathy Diabetes. and 2014;5(3):393-8. https://dx.doi.org/10.4239/wjd.v5.i3.393. $\uparrow$ Ver página 3

[7] Peti-Peterdi J, Kang JJ, Toma I. Activation of the renal renin-angiotensin system in diabetes - New concepts. Nephrol Dial Transplant. 2008;23(10):3047-9. https://dx.doi.org/10.1093/ndt/ gfn377. $\uparrow$ Ver página 3

[8] Musante L, Tataruch D, Gu D, Liu X, Forsblom C, Groop PH, et al. Proteases and protease inhibitors of urinary extracellular vesicles in diabetic nephropathy. J Diabetes Res.

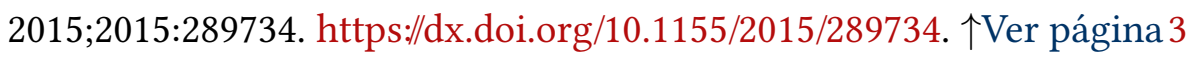


[9] Livingstone SJ, Levin D, Looker HC, Lindsay RS, Wild SH, Joss N, et al. Estimated life expectancy in a scottish cohort with type 1 diabetes, 2008- 2010. JAMA. 2015;313(1):37-44. https:/dx.doi.org/10.1001/jama.2014.16425. 个Ver página 4, 7

[10] Tonneijck L, Muskiet MHA, Smits MM, van Bommel EJ, Heerspink HJ, et al. Glomerular hyperfiltration in diabetes: Mechanisms, clinical significance, and treatment. J Am Soc

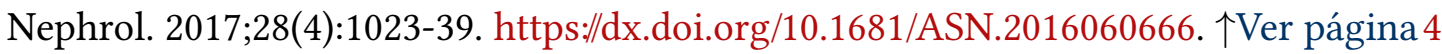

[11] Porrini E, Ruggenenti P, Luis-Lima S, Carrara F, Jiménez A, de Vries APJ, et al. Estimated GFR: time for a critical appraisal. Nat Rev Nephrol. 2019;15(3):177-90. https:/dx.doi.org/10. 1038/s41581-018-0080-9. 个Ver página 4

[12] Mansour I, Thajudeen B. Overview of diabetic nephropathy. En: Bakris GL, Hahr A, Khardori R, Koya D, Molitch M, Prischl FC, et al., editores. Managing Diabetic Nephropathies in Clinical Practice. Springer International Publishing Switzerland; 2017. https://x.doi.org/ 10.1007/978-3-319-08873-0. 个Ver página 4

[13] Berhane AM, Weil EJ, Knowler WC, Nelson RG, Hanson RL. Albuminuria and estimated glomerular filtration rate as predictors of diabetic end-stage renal disease and death. Clin J Am Soc Nephrol. 2011;6(10):2444-51. https://dx.doi.org/10.2215/CJN.00580111. $\uparrow$ Ver página 4

[14] Zeni L, Norden AGW, Cancarini G, Unwin RJ. A more tubulocentric view of diabetic kidney disease. J Nephrol. 2017;30(6):701-17. https:/dx.doi.org/10.1007/s40620-017-0423-9. 个Ver página 5

[15] Ruggenenti P, Porrini EL, Gaspari F, Motterlini N, Cannata A, Carrara F, et al. Glomerular hyperfiltration and renal disease progression in type 2 diabetes. Diabetes Care. 2012;35(10):2061-8. https://dx.doi.org/10.2337/dc11-2189. 个Ver página 5

[16] Schalkwijk CG, Stehouwer CDA. Vascular complications in diabetes mellitus: The role of endothelial dysfunction. Clin Sci (Lond). 2005;109(2):143-59. https://x.doi.org/10.1042/ CS20050025. $\uparrow$ Ver página 5

[17] Toth-Manikowski S, Atta MG. Diabetic kidney disease: Pathophysiology and therapeutic

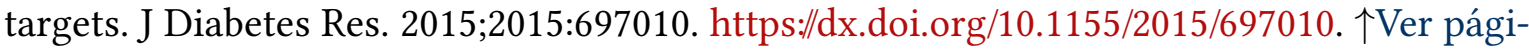
na 6

[18] Restrepo-Valencia CA, Buitrago-Villa CA, Torres-Saltarín JJ, Serna-Flórez J, editores. Nefrología básica 2. Bogotá D.C.: Asociacxión Colombiana de Nefrología e Hipertención Arterial; 2019. $\uparrow$ Ver página 6 
[19] American Diabetes Association. 11. Microvascular complications and foot care: Standards of Medical Care in Diabete-2020. Diabetes Care. 2020;43(Suppl 1):S135-S151. https:/dx.doi. org/10.2337/dc20-S011. $\uparrow$ Ver página 7

[20] Tuttle KR, Bakris GL, Bilous RW, Chiang JL, de Boer IH, Goldstein-Fuchs J, et al. Diabetic kidney disease: A report from an ADA consensus conference. Diabetes Care. 2014;37(10):2864-83. https:/dx.doi.org/10.2337/dc14-1296. 个Ver página 8

[21] National Kidney Foundation. KDOQI clinical practice guideline for diabetes and CKD: 2012 update. Am J Kidney Dis. 2012;60(5):850-86. https:/dx.doi.org/10.1053/j.ajkd.2012.07. 005. $\uparrow$ Ver página 8

[22] Wang L, Wu J, Cheng JF, Liu XY, Ma F, Guo LH, et al. Diagnostic value of quantitative contrast-enhanced ultrasound (CEUS) for early detection of renal hyperperfusion in diabetic kidney disease. J Nephrol. 2015;28(6):669- 78. https://x.doi.org/10.1007/ s40620-015-0183-3. 个Ver página 8

[23] Filip S, Zoidakis J, Vlahou A, Mischak H. Advances in urinary proteome analysis and applications in systems biology. Bioanalysis. 2014;6(19):2549-69. https://x.doi.org/10.4155/ bio.14.210. $\uparrow$ Ver página 8,10

[24] Merchant ML, Klein JB. Proteomic Discovery of Diabetic Nephropathy Biomarkers. Adv Chronic Kidney Dis. 2010;17(6):480-6. https:/dx.doi.org/10.1053/j.ackd.2010.09.001. $\uparrow$ Ver página 8

[25] Moresco RN, Sangoi MB, De Carvalho JAM, Tatsch E, Bochi GV. Diabetic nephropathy: Traditional to proteomic markers. Clin Chim Acta. 2013;421:17- 30. https:/dx.doi.org/10. 1016/j.cca.2013.02.019. $\uparrow$ Ver página 8

[26] $\mathrm{Wu}$ J, Chen YD, Gu W. Urinary proteomics as a novel tool for biomarker discovery in kidney diseases. J Zhejiang Univ Sci B. 2010;11(4):227-37. https:/dx.doi.org/10.1631/jzus. B0900327. $\uparrow$ Ver página 9

[27] Gao YH. Urine-an untapped goldmine for biomarker discovery? Sci China Life Sci. 2013;56(12):1145-6. https:/dx.doi.org/10.1007/s11427-013-4574-1. 个Ver página 9

[28] Weissinger EM, Wittke S, Kaiser T, Haller H, Bartel S, Krebs R, et al. Proteomic patterns established with capillary electrophoresis and mass spectrometry for diagnostic purposes. Kidney Int. 2004;65(6):2426-34. https:/dx.doi.org/10.1111/j.1523-1755.2004.00659.x. 个Ver página 9 
[29] Haubitz M, Wittke S, Weissinger EM, Walden M, Rupprecht HD, Floege J, et al. Urine protein patterns can serve as diagnostic tools in patients with IgA nephropathy. Kidney Int. 2005;67(6):2313-20. https:/dx.doi.org/10.1111/j.1523-1755.2005.00335.x. $\uparrow$ Ver página 9

[30] Julian BA, Wittke S, Novak J, Good DM, Coon JJ, Kellmann M, et al. Electrophoretic methods for analysis of urinary polypeptides in $\lg \mathrm{A}$ - associated renal diseases. Electrophoresis. 2007;28(23):4469-83. https://dx.doi.org/10.1002/elps.200700237. 个Ver página 9

[31] Rossing K, Mischak H, Dakna M, Zürbig P, Novak J, Julian BA, et al. Urinary proteomics in diabetes and CKD. J Am Soc Nephrol. 2008;1283-90. https:/dx.doi.org/10.1681/ ASN.2007091025. $\uparrow$ Ver página 9

[32] Haubitz M, Good DM, Woywodt A, Haller H, Rupprecht H, Theodorescu D,et al. Identification and validation of urinary biomarkers for differential diagnosis and evaluation of therapeutic intervention in anti-neutrophil cytoplasmic antibody-associated vasculitis. Mol Cell Proteomics. 2009;8(10):2296-307. https:/dx.doi.org/10.1074/mcp.M800529-MCP200. $\uparrow$ Ver página 9

[33] Zou L, Sun W. Human urine proteome: A powerful source for clinical research. Adv Exp Med Biol. 2015;845:31-42. https:/dx.doi.org/10.1007/978-94-017-9523-4_4. 个Ver página 9

[34] Jun M, Ohkuma T, Zoungas S, Colagiuri S, Mancia G, Marre M, et al. Changes in albuminuria and the risk of major clinical outcomes in diabetes: Results from ADVANCE-ON.

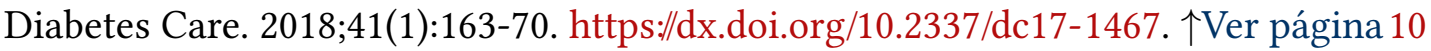

[35] Thongboonkerd V. Study of diabetic nephropathy in the proteomic era. Contrib Nephrol. 2011;170:172-83. https://dx.doi.org/10.1159/000325657. $\uparrow$ Ver página 10

[36] Zürbig P, Jerums G, Hovind P, Macisaac RJ, Mischak H, Nielsen SE, et al. Urinary proteomics for early diagnosis in diabetic nephropathy. Diabetes. 2012;61(12):3304-13. https:// dx.doi.org/10.2337/db12-0348. $\uparrow$ Ver página 11, 12

[37] Papale M, Di Paolo S, Magistroni R, et al. Urine proteome analysis may allow noninvasive differential diagnosis of diabetic nephropathy. Diabetes Care. Published online 2010. https:

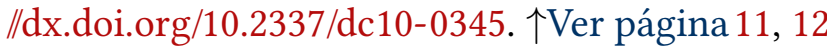

[38] Roscioni SS, De Zeeuw D, Hellemons ME, Mischak H, Zürbig P, Bakker SJ, et al. A urinary peptide biomarker set predicts worsening of albuminuria in type 2 diabetes mellitus. Diabetologia. 2013;56(2):259-67. https:/dx.doi.org/10.1007/s00125-012-2755-2. 个Ver página 11, 12

[39] Nielsen SE, Schjoedt KJ, Astrup AS, Tarnow L, Lajer M, Hansen PR, et al. Neutrophil gelatinase-associated lipocalin (NGAL) and kidney injury molecule 1 (KIM1) in patients 
with diabetic nephropathy: A cross-sectional study and the effects of lisinopril. Diabet Med.

https:/dx.doi.org/10.1111/j.1464-5491.2010.03083.x. 个Ver página 12 